\title{
La movilidad socio-espacial desde la teoría de Pierre Bourdieu: capital de motilidad, campo de movilidad y habitus ambulante $^{1}$
}

Socio-spatial Mobility from Pierre Bourdieu's Theory: Capital of Motility, Field of Mobility and Mobile Habitus

\section{A mobilidade socioespacial desde a teoria de Pierre Bourdieu: capital de motilidade, cam- po de mobilidade e habitus ambulante}

\author{
Francisco Adolfo García Jerez ${ }^{2}$
}

Profesor e investigador de la Universidad del Valle, Cali, Colombia

adolfo.garcia@correounivalle.edu.co

Recibido: 10/06/2015

Aprobado: 06/05/2016

1 Este artículo es producto de la investigación titulada "Planificación urbanística, hábitos y representaciones sobre la movilidad socio-espacial: un análisis de la motilidad en dos barrios de Cali" (C.I. 6166), la cual fue financiada con recursos procedentes de la Convocatoria interna de la Universidad del Valle (Cali, Colombia) para el período de julio de 2014 a julio de 2015 . Es por ello que agradezco a la Universidad del Valle por la financiación recibida. También extiendo dicho agradecimiento a los evaluadores externos por sus valiosos e interesantes comentarios.

2 Doctor en Antropología Social. 


\title{
Resumen
}

En las últimas décadas se ha suscitado un interés sociológico por los medios de transporte y en general por la ontología de la movilidad. Este interés ha estado motivado, entre otras cuestiones, por el desarrollo tecnológico que ha transformado radicalmente los medios de transportes reestructurando a su vez las nociones de espacio y tiempo, así como las mismas lógicas de movilidad espacial. Pensamos que en la tarea de analizar dichos cambios sociales la sociología ofrece suficiente bagaje e instrumentos teórico-metodológicos. En este sentido, este artículo desea contribuir a esos debates planteando una propuesta teórica que desde el estructuralismo-constructivista de Pierre Bourdieu aborde la cuestión de la movilidad socio-espacial. Para ello hemos aplicado las nociones de capital de motilidad, campo de movilidad y habitus ambulante.

Palabras clave: movilidad socio-espacial; campo de movilidad; capital de motilidad; habitus ambulante; sociología.

\begin{abstract}
In the last decades a sociological interest has been raised on means of transportation and in general on ontology of mobility. This interest has been generated, among others, due to technological development which has transformed deeply the means of transportation and, with it, has restructured notions such as space and time as well as the logics of spatial mobility. We think that in this task, sociology offers enough background and theoretical and methodological instruments in order to explain these social changes. In this sense, this article would wish to contribute to these debates rising a theoretical propose that, from constructivist-structuralism of Pierre Bourdieu, deal with the issue of socio-spatial mobility. For that, we have applied the notions of capital of motility, field of mobility and mobile habitus.
\end{abstract}

Keywords: socio-spatial mobility; field of mobility; capital of motility; mobile habitus; sociology.

\section{Resumo}

Nas últimas décadas tem emergido um interesse sociológico pelos meios de transporte e, em geral, pela ontologia da mobilidade. Esse interesse tem estado motivado, por exemplo, pelo desenvolvimento tecnológico, que vem transformando os meios de transportes, recompondo as noções de espaço, tempo e as lógicas de mobilidade espacial. Na tarefa de analisar essas mudanças sociais, acreditamos que a sociologia fornece suficiente bagagem e instrumentos teóricos e metodológicos. Nessa ordem, este artigo busca contribuir nos debates, apresentando uma proposta teórica, baseada no estruturalismo-construtivista de Pierre Bourdieu para abordar a questão da mobilidade socioespacial. Assim, aplicamos as noções de capital de motilidade, campo de mobilidade e habitus ambulante.

Palavras-chave: mobilidade socioespacial; campo de mobilidade; capital de motilidade; habitus ambulante; sociologia.

Este trabajo está bajo la licencia Creative Commons Attribution 3.0

¿Cómo citar este artículo? / How to quote this article?

García-Jerez, Francisco «La movilidad socio-espacial desde la teoría de Pierre Bourdieu: capital de motilidad, campo de movilidad y habitus ambulante». Sociedad y economía, No. 31 (julio - diciembre 2016): 15-32. 


\section{Introducción}

Como señalan Urry (2000) y Bericat (1994), desde la década de 1990 la movilidad ha ido adquiriendo una importancia relevante en orden a comprender los cambios sociales más generales. Ambos autores sitúan la movilidad socio-espacial y el viaje como aspectos centrales de nuestra sociedad por cuanto están reconfigurando las percepciones sobre el espacio y el tiempo, así como las relaciones y prácticas sociales. Como consecuencia de las nuevas tecnologías aplicadas tanto a las redes telemáticas como a los medios de transporte, se ha constituido una nueva simultaneidad, lo que significa cambios sustanciales en hacer política, en el ejercicio del poder y la resistencia a él, pero también en los modos de percibir y organizar la vida cotidiana, puesto que como bien apunta David Harvey (1998) el espacio se ha ido acortando en términos temporales.

Frente a estos cambios en la movilidad espacial y su correlato tecnológico, las principales aportaciones de la sociología al campo de análisis del transporte y la movilidad³, según Almaraz et al. (2010), podrían resumirse en tres grandes bloques. Un primero que analiza el papel desempeñado por el sistema de transporte en la configuración urbana. Fue Manuel Castells (1974) quien, a partir de su teoría de la circulación, afirmaba que cuanta más complejidad caracterizaba al ámbito urbano más importante resultaban las conexiones internas. Para dicho autor esa red de transporte debía situarse dentro de una teoría del intercambio entre los componentes del sistema urbano en el que la naturaleza del propio objeto explicaba el modo de su propia circulación. Un segundo bloque englobaría los aspectos sociales de la demanda del transporte. Esta premisa permite explorar los intereses y necesidades de los diferentes sectores sociales y adaptar la oferta a esas necesidades. Y una tercera perspectiva que, tomando como partida los trabajos de Urry (2004; 2000) y Sheller (2004), profundiza en el fenómeno de la motorización de las sociedades actuales y sus consecuencias sociales. Esto conlleva, según Soerensen (mencionado en Jenssen 1999), a indagar sobre aspectos relacionados con la vida diaria, los estilos de vida y la cultura cotidiana de movilidad de los individuos, ya que no solo es pertinente atender a las lógicas racionales y economicistas de las prácticas socio-espaciales, sino también concentrarse en la dimensión afectivo-emocional que las envuelven.

Con respecto a esta última cuestión han sido Canzler, Kaufmann y Kesselring (2008), Flamm y Kaufmann (2006) y Kaufmann, Bergman y Joye (2004) quienes reflexionando acerca de dicho fenómeno han introducido una nueva categoría con la que poder analizar de un modo holístico la movilidad socio-espacial de grupos e individuos. Esta es la de la motility (motilidad). Por ella podría entenderse, tal y como bien la definen Kaufmann, Bergman y Joye (2004), el grado de movilidad no solo real sino también potencial de los individuos, grupos u otras entidades y cómo esta es transformada en desplazamientos a partir de su apropiación. Y es esta categoría la que nos invita a elaborar un marco teórico que

3 Es imprescindible mencionar el trabajo pionero de Charles Horton Colley titulado The theory of transportation publicado a finales del siglo XIX, en el que analiza el transporte y la movilidad desde un enfoque holístico ensamblando cuestiones relativas al medio físico con la organización social, militar, económica, urbana y política de las sociedades. 
permita situar a la movilidad espacial como su objeto de análisis y develar, así como entender, sus lógicas sociales.

De este modo, el objetivo de este artículo es presentar una propuesta teórica de análisis de la movilidad socio-espacial que en esta ocasión se fundamentará en los planteamientos del estructuralismo-constructivista del sociólogo francés Pierre Bourdieu. Con tal fin se ha dividido en cinco apartados: un primero en el que se presentará una breve aproximación a las ideas que sustentan la teoría bourdieuiana; le seguirán un segundo y tercero en los que se aplicarán algunos de esos conceptos a la categoría de motilidad, un cuarto donde se desarrollará la noción de habitus ambulante y se cerrará el artículo con un quinto apartado donde se enunciarán unas breves conclusiones.

\section{Una aproximación al estructuralismo- constructivista de Pierre Bourdieu}

Pensamos que la perspectiva estructuralista-constructivista puede ser un sugerente marco teórico para coadyuvar en el objetivo de evaluar el grado de motilidad o, más específicamente, los motivos, razones o lógicas subyacentes a los desplazamientos realizados por los agentes en la vida diaria. Ello no desde una perspectiva de la elección racional o desde un ethos psicológico, sino atendiendo a las estructuras externas que condicionan esas prácticas ambulantes, así como las estructuras internas de cada agente o clase en la articulación de estrategias para el despliegue de sus desplazamientos. Es decir, y como se analizará a continuación, esta perspectiva nos obliga a entender el grado de "acceso" como un aspecto externo condicionante de las prácticas ambulantes, así como las "competencias" y la "apropiación" como guías de esas mismas prácticas, pero con un componente social o gregario. Estos tres componentes forman parte de lo que Canzler, Kaufmann y Kesselring (2008) denominan "motilidad"; es decir, la capacidad de un actor de moverse social y espacialmente. Por consiguiente, la motilidad es cómo un individuo o grupo hace suyo el campo de las posibilidades de movimiento y las utilizan. Asimismo, y dado que el grado de acceso y parte de las competencias no son desplegadas equitativamente, su puesta en juego generará tensiones sociales y escenarios conflictuales.

De este modo, si deseamos entender la categoría de motilidad es necesario aplicar una serie de conceptos sugeridos por Pierre Bourdieu tales como campo, capital y habitus desde una perspectiva relacional en la que se entrecrucen "las estructuras objetivas (la de los campos sociales) y las estructuras incorporadas (las de los habitus)" $(1999,8)$. Dicho autor entiende que todo comportamiento humano posee una dimensión ontológicamente social que debe explicarse desde lo social, por cuanto "los agentes no llevan a cabo actos gratuitos" $(1999,140)$. Cualquier decisión de desplazamiento, de viaje, trayectoria y modos de realizarlos, aunque parezcan efectos de impulsos inconscientes, poseen una lógica social cuya coherencia es producto de la existencia en el comportamiento humano de un interés interiorizado por el cual se actúa o se participa en el juego social. Para Pierre Bourdieu ese interés podría denominarse "libido" o "illusio": "La illusio es el hecho de estar en el juego, cogido por el juego, de creer que el juego merece la pena jugar (...) por lo tanto reconocer que el juego merece ser 
jugado y que los envites que se engendran en y por el hecho de jugarlo merecen seguirse; significa reconocer el juego y reconocer los envites" (1999, 141). Ese interés, o mejor, la diversidad de intereses será lo que genere un espacio de luchas y conflictos. Ahora bien, para que esto suceda es necesaria la existencia de posiciones diferentes que, en función de esas relaciones jerárquicas, doten al agente de mayor o menor proximidad a aquel bien codiciado por el que se está en disputa.

Así pues, para que tengan lugar estas interrelaciones, y en especial para entender y analizar el propio juego, es necesario recuperar las nociones dadas por el propio sociólogo francés de campo, capital y habitus. La primera de ellas, la de campo social, es definida por Bourdieu como "...espacios de juego históricamente constituidos con sus instituciones específicas y sus leyes de funcionamiento propias" (Bourdieu, mencionado en Gutiérrez 2005, 31). Tomando como partida la síntesis realizada por Gutiérrez (2005), las leyes que caracterizan a un campo podrían ser las siguientes: en primer lugar, los campos se presentan como "sistemas de posiciones y de relaciones entre posiciones" (Costa, mencionado en Gutiérrez 2005, 31), o en palabras del propio Bourdieu: "conjunto de posiciones distintas y coexistentes, externas unas a otras, definidas en relación unas de otras, por su exterioridad mutua y por relaciones de proximidad, vecindad o de alejamiento y asimismo por relaciones de orden como por encima, por debajo y entre" $(1999,16)$. En segundo lugar, un campo se define, entre otros aspectos, por lo que está en juego (en jeu) y los intereses específicos del mismo. De este modo, para que funcione un campo es necesario que haya algo en juego y gente dispuesta a jugar. Por tanto, los agentes en la medida en que están interesados en satisfacer sus intereses decidirán involucrarse en el juego: "Es evidente que, entre las personas que ocupan posiciones opuestas en un campo y que parecen radicalmente opuestas en todo, existe un acuerdo oculto y tácito sobre el hecho de que vale la pena luchar por cosas que están en juego en ese campo" $(1999,143)$. En tercer lugar, la estructura del campo es un estado de la distribución en un momento dado del tiempo, del capital específico que está en juego. Esa distribución estará supeditada al volumen del capital acumulado, pero también a la jerarquía del valor de los capitales obtenidos o su posición en la estructura del capital; por tanto, "los agentes están distribuidos según el volumen de capital que posee bajo sus diferentes especies y en la segunda dimensión según la estructura de su capital, es decir según el peso relativo de las diferentes especies de capital" $(1999,18)$. Asimismo, esa estructura también es un estado de relaciones de fuerza entre los agentes que están comprometidos en el juego, lo que la convierte en un campo de luchas en la que los agentes tenderán a conservar o transformar sus capitales en pro de mantener o cambiar la definición del propio juego. Y en cuarto lugar, y precisamente por estar sometido a una permanente revisión, los campos poseen un carácter dinámico e histórico; o como bien lo manifiesta el propio Pierre Bourdieu:

El campo (es decir el espacio de juego, las reglas del juego, las apuestas, etc.) se ofrece claramente como lo que es, una construcción social arbitraria y artificial, un artefacto que se invoca como tal en todo lo que define su autonomía, reglas explícitas y específicas, espacio y tiempo estrictamente delimitados y extraordinarios, y la entrada en el juego adquiere la forma de un cuasi contrato que a veces es explícitamente evocado $(2008,108)$. 
En ese proceso Gutiérrez (2005), siguiendo a Bourdieu, apunta que para la consolidación de un campo social es imprescindible la configuración de un mercado específico relacionado con ese mismo campo. En este, al igual que cualquier mercado, aparecen agentes que asumen el papel de productor del bien puesto en circulación, de consumidores y de intermediarios, así como instancias de consagración. Esto es posible gracias a la activación de dos dispositivos: la monopolización de los bienes por unos y su desposesión de otros a partir de un proceso de reconocimiento y legitimación. Por tanto, en este tipo de mercado, $\mathrm{y}$ al igual que el puramente comercial, se elaboran productos en función de las demandas, gustos o distinciones dependiendo a su vez de dos aspectos: de un lado, de la condición y de la clase y, de otro, de la competencia entre oferentes. Esto significa, en palabras de Gutiérrez, que la lógica del campo de la producción y la lógica del campo de consumo han de coordinarse objetivamente a partir del "principio de la homología funcional y estructural" $(2005,59)$. Este principio podría resumirse del siguiente modo: todos los campos se organizan bajo una misma lógica basada en una distribución desigual de un capital, estableciéndose relaciones jerárquicas y de dominación entre los agentes en función del volumen y jerarquía de los capitales acumulados. Asimismo, los campos poseen una autonomía relativa por cuanto todos ellos forman parte de un espacio social global.

Como ya se mencionó, para la existencia de un campo es imprescindible que se ponga un bien en juego. Ese bien ha de ser interpretado por los agentes como un recurso escaso, limitado y por consiguiente codiciado, es decir un capital, el cual podría definirse como "un conjunto de bienes acumulados que se producen, se distribuyen, se consumen, se invierten, se pierden" (Costa, mencionado en Gutiérrez 2005, 34). De algún modo, este es el elemento central del conflicto social y del interés de los agentes, ya que su tenencia sitúa a estos en una posición determinada en el propio campo. En palabras de Pierre Bourdieu: "la posición ocupada en el espacio social, es decir en la estructura de la distribución de las diferentes especies de capital, que asimismo son armas, ordena las representaciones de este espacio y las tomas de posición en las luchas para conservarlo o transformarlo" $(1999,25)$. Sin embargo, como ya se expresó, la posición del agente no solo está mediada por el volumen, sino también por la estructura de los capitales que cada cual haya acumulado.

En suma, existe un campo en el que se pone en juego un capital que dependiendo de su carácter definirá la naturaleza del propio campo. Ese capital -mejor, su acumulación, volumen y jerarquía- así como su articulación con los otros capitales, situará al agente que lo ostenta en una determinada posición. Esa posición no solo le proporcionará un nivel de fuerza en las relaciones con los otros agentes, sino que definirá su modo de ser y estar; o dicho con las palabras del propio Bourdieu: "A cada clase de posición corresponde una clase de habitus (o de aficiones)..." (1999, 19). Ese habitus no es baladí por cuanto le dota al agente y a sus pares de una mirada sobre la realidad, siendo esta precisamente una de sus funciones primordiales. Pierre Bourdieu se refiere a este concepto del siguiente modo: "Los habitus son principios generadores de prácticas distintas y distintivas (...) pero también son esquemas clasificatorios, principios de clasificación, principios de visión y división, aficiones, diferentes" $(1999,20)$. Por tanto, alinea 
las prácticas entre aquellos que comparten una misma posición; de ahí que los agentes deberían hacer aquello que se espera que realicen, pero también les proporciona un sentido de la vida, de su propia práctica y una representación de la realidad social. Además de proporcionar al agente una guía, los habitus también con su materialización permiten establecer y que sean reconocidas las diferentes posiciones $\mathrm{y}$, con ello, los diversos grupos que participan en uno o en varios campos, puesto que "como las posiciones de las que son productos, los habitus se diferencian; pero asimismo son diferenciantes. Distintos y distinguidos, también llevan a cabo distinciones" (1999, 19-20).

\section{El campo de la movilidad}

¿Podríamos referir a la existencia de un campo de la movilidad socio-espacial?; si fuera así, ¿qué características diferenciales tendría?, ¿cuál sería su capital?, ¿podría germinar un habitus particular relacionado con la motilidad?

En mi opinión, la movilidad es constitutiva de un campo propio, lo que no es óbice para que existan intersecciones y yuxtaposiciones con otros campos. Sin embargo, los desplazamientos, los trayectos diarios o excepcionales, los viajes y todo lo concerniente a la movilidad pueden ser interpretados del mismo modo que los componentes o elementos religiosos o culturales y su incardinación en un espacio de relaciones y luchas. De este modo, el campo de la movilidad ${ }^{4}$, al igual que los anteriormente aludidos, sería un sistema basado en relaciones sociales jerárquicas y de dominaciones entre posiciones. En este sistema aparecería una serie de agentes con posiciones bien definidas, los cuales estarían divididos en clases con un desigual acceso a la movilidad y caracterizados por diferentes gustos y modos de distinción en cuanto a sus potenciales o reales desplazamientos. Este campo y sus tensiones quedarían ilustrados, por ejemplo, en el trabajo de Jenssen (1999), en el cual se tipifican dos grandes grupos de usuarios de transporte en Dinamarca, cada uno con sus subtipos: los conductores de vehículos (que englobarían a los conductores apasionados, los diarios y los de tiempo de ocio) y los ciclistas y usuarios de trasporte público (los cuales se dividen en usuarios "de corazón", por conveniencia y por necesidad). Cada uno de estos tipos posee, además de unas prácticas de movilidad, una visión sobre la idea del automóvil asociado con la independencia, libertad y medio ambiente. Lo interesante a resaltar es que la edad, la tenencia de hijos, los ingresos económicos y la conciencia ambiental son las variables que cruzadas explican el perfil de cada uno de los grupos y sus comportamientos ambulantes. En definitiva, cada grupo dispone de un habitus particular que estará conformado por la posibilidad de elección -no siempre en las mismas condiciones- dentro de

4 En mi opinión habría que diferenciar el campo de la movilidad del campo del transporte. Como se expondrá más adelante, este último girará en torno a la industria y a las políticas públicas del transporte y en los que podrían resaltarse entre sus principales agentes las empresas de transporte, los lobbies de transportistas y las grandes constructoras que acumulan capital económico, social y simbólico; así como las administraciones públicas, las cuales durante algunas décadas han sido las instituciones intermediarias en la provisión de este bien (es decir, el garantista en el acceso a la movilidad espacial). 
un marco estructurado5, pero también de un sentido moral de lo que implica determinadas prácticas de movilidad para el conjunto de la sociedad. Por tanto, y siguiendo la noción de illusio de Pierre Bourdieu (1999; 2008), todos los agentes serían conscientes de la existencia de un bien que por su valor es codiciado y que caracterizará y definirá el campo de la movilidad.

Asimismo, y al igual que otros campos sociales, este, el de la movilidad, también está sometido a la historicidad; es decir, a cambios tanto en su carácter ontológico como en los agentes que lo componen. Pensemos en los cambios ocurridos en los albores del siglo XIX, cuando entre las clases burguesas europeas comenzaron a revalorizar la práctica peatonal tanto en el ámbito de lo rural (el pasear) como en lo urbano con la aparición de la actitud del flâneur (el caminar o flâner) a partir del descubrimiento de los estímulos sensoriales emitidos por la nueva metrópolis (Marqués 2015). O con la invención del automóvil y lo que devino en cuanto a la aparición de una nueva dimensión que se sumaría al eje espacio-tiempo: la de la velocidad, la cual modificaría la concepción de la movilidad y su práctica cotidiana 6 . O el último de los cambios: el que sitúa la movilidad como un aspecto prioritario en las políticas públicas. Esta prioridad se debe no solo a su naturaleza estratégica relacionada con lo puramente económico, por cuanto su configuración regula la puesta en circulación de productos y personas, sino que también forma parte de uno de los rasgos posestructuralistas de nuestra época enraizado en la noción formulada por Ulrich Beck de "la sociedad del riesgo" (1999)7. En efecto, en esa nueva conciencia el transporte motorizado se ha situado como uno de los principales factores de esa acción antrópica generadora de efectos perniciosos tanto para el medio ambiente como para el ser humano, lo que ha conducido a que instituciones públicas, organizaciones y asociaciones estén planteando otras lógicas alternativas de movilidad. Un ejemplo de ello es la aparición, articulación y revigorización del critical mass (Furness 2007; Blickstein y Hanson 2001), cuyo objetivo es reclamar la relevancia de la bicicleta como medio de transporte ecológica y socialmente sostenible.

Así pues, esas continuas resemantizaciones de la naturaleza del campo de la movilidad apuntan, de un lado, a su carácter histórico y dinámico, y, de otro, a la observación de intereses contrapuestos que dan lugar a luchas por modificar tanto el reparto del capital puesto en juego como también en redefinir la propia

5 En este mismo sentido habría que destacar la investigación realizada por Ureta (2009), en la cual describe los motivos y percepciones existentes en la adquisición de un automóvil por un grupo de familias de pocos recursos económicos en la ciudad de Santiago de Chile, así como los cambios que produce en la práctica de su movilidad, gustos y hábitos.

6 De hecho, en opinión del filósofo francés Paul Virilio (1997), el aumento de la velocidad, ocasionado por la aparición de nuevos dispositivos de movilidad como el cada vez más veloz y hermético automóvil, los trenes de alta velocidad y los aviones (junto con el internet), estarían provocando una "contaminación dromosférica"; es decir, como consecuencia del uso de estos dispositivos los usuarios estarían reduciendo su experiencia sensible con el entorno.

7 Kesselring (2008) es uno de los autores que mejor ha retomado esta noción y la ha aplicado al ámbito de las movilidades socio-espaciales. Dicho autor parte de la premisa de la existencia de un contexto social cada vez más inestable, contingente, impredecible y que ha sido reflexivamente procesado por los individuos, por lo que el sujeto ha comenzado a desplegar una diversidad de estrategias y técnicas de movilidad a fin de amoldarse a esa situación. A esta competencia, la cual podría interpretarse como un verdadero dispositivo de adaptación, el autor lo denomina "mobility management". 
naturaleza del campo. Sin embargo, estos cambios no solo son producidos en y por el campo de la movilidad, teniendo en consideración "la relativa autonomía de los campos" y "la pluridimensionalidad del espacio social" enunciadas por Pierre Bourdieu, el campo de la movilidad estaría atravesado a su vez por otros muchos espacios. Entre ellos destaca sobremanera el del transporte, el cual tendría como razón de ser los procesos industriales, los proveedores de movilidad y las políticas públicas relativas a ello. En ese espacio operarían las multinacionales del ramo y las alianzas entre agentes públicos y privados; pero también los tratados de libre comercio, como los sellados entre Colombia y México, Estados Unidos o Corea del Sur ${ }^{8}$ así como los discursos hegemónicos puestos en circulación cuyo fin es legitimar las prácticas de movilidad con base en la idea de modernidad o, más recientemente, en la de la sostenibilidad, y que en ocasiones han podido ocultar otras intenciones, tal como las evidenciadas por Adam Greenfield (2013) en relación con los efectos privatizadores en la aplicación de las llamadas Smart City.

\section{El capital de motilidad}

Elliott y Urry (2010) se referían a la movilidad y a su posible encuadre teórico enfatizando la importancia de las relaciones sociales en red y cómo estas están estrechamente vinculadas a la capacidad de movilidad, convirtiéndose en una variable básica del "campo de las movilidades". Teniendo en consideración dicha premisa, podría afirmarse que han sido dos de los primeros autores en contemplar que la movilidad socio-espacial es constitutiva de un capital. Parten de la premisa de que, vinculado con la movilidad socio-espacial, existe un particular tipo de capital que se pone en juego. Este estaría definido no tanto por los componentes de acceso, competencias y apropiación, sino por los vínculos sociales que el individuo o grupo establece y que facilitarían la propia práctica de la movilidad. Ese capital daría lugar, por tanto, al campo de la movilidad. Sin embargo, en nuestra opinión, ese capital de red formaría parte de lo descrito por Pierre Bourdieu como capital social. Recordemos que por este el sociólogo francés entendía lo siguiente:

[El] conjunto de los recursos actuales o potenciales que están ligados a la posesión de una red duradera de relaciones más o menos institucionalizadas de interconocimiento y de inter-reconocimiento; o, en otros términos, a la pertenencia a un grupo, como conjunto de agentes que no sólo están dotados de propiedades comunes (susceptibles de ser percibidas por el observador, por los otros o por ellos mismos), sino que también están unidos por lazos permanentes y útiles (Bourdieu, mencionado en Gutiérrez 2005, 37-38).

8 Dichos tratados posibilitan en los mercados receptores el asentamiento de empresas y firmas dedicadas a la venta de automóviles conllevando una mayor oferta y variedad de precios que, junto a las facilidades financieras de adquisición de créditos para la compra de un vehículo y el subsidio al petróleo, están incentivando el aumento del parque automotor. Según las estimaciones de Acevedo et al. (2009) hasta el 2040 se producirá en Colombia un muy fuerte incremento de la tasa de motorización, puesto que se multiplicarían por 3 las cifras actuales de automóviles (de 3 millones en el 2009 a 10,4 en el 2040) y por 5,5 el de motos (pasando de 3,3 millones a 13 millones), aunque todavía esa tasa de motorización estaría por debajo de las de los otros países de la región. 
De este modo, dada la naturaleza del denominado capital de red este no debería constituir per se un capital particular ni por tanto definir un nuevo campo sino, al contrario, integrarse en el ya delimitado campo de lo social. En efecto, a diferencia de esta propuesta mi tesis se fundamenta en la existencia de un capital de motilidad como tal que directamente se circunscribe a la posibilidad real o potencial de movilizarse espacialmente. Su desigual distribución delimita la existencia de un espacio de luchas, el cual, como ya se expuso, podríamos denominarlo campo de la movilidad. En él se pondría en juego la posibilidad real o potencial de desplazarse y lo que ello implica. Cierto es que el juego y las posiciones de los agentes en ese campo estarían condicionados entre otros capitales por el social. Es por ello que la configuración de redes sociales de vínculos sostenidos, densos, confiables e intensos puede revelarse un aspecto central de este campo, aunque en ningún caso estas redes deberían ser interpretadas como determinantes en la adquisición de dicho capital. Así como en el campo económico se puede ostentar una muy buena posición y aumentar el capital económico sin necesidad de poseer un gran volumen de capital social (pensemos en la acumulación de capital económico obtenida fundamentalmente por medio de la inversión especulativa, o la de las grandes fortunas heredadas), en el campo de movilidad un individuo puede tener la posibilidad de movilizarse sin necesidad de esos lazos sociales. Esto no es óbice para que, como ya he manifestado, la "relativa autonomía de los campos" permita la yuxtaposición de los capitales, su interacción y retroalimentación.

Otra de las propuestas que mejor incardina la noción de capital y la de movilidad es la formulada por Jouffe. Dicho autor concibe la movilidad como un campo constituido por un capital de segundo orden y marginal, en definitiva un "para-capital" que estaría compuesto "por otros capitales y puesto al servicio de la acumulación de estos, no al revés, o sea, un recurso susceptible de acumulación pero no objeto de lucha por sí" (2011, 89). Este "para-capital" estaría configurado, por tanto, por tres tipos de capitales: el social, el económico y el de acceso, los cuales reportan al sujeto una posición con respecto a su grado de movilidad. Sin embargo, Jouffe incluye una nueva dimensión en orden a resituar la movilidad, puesto que lo económico, lo social y el acceso estarían mediados, según dicho autor, por el hábitat. Su premisa sitúa la centralidad y el grado de infraestructura que posee el barrio y la vivienda -ambas como expresiones de hábitat- como las variables independientes para entender y explicar las lógicas de desplazamientos reales o potenciales de los individuos. Su aportación es de gran interés en la medida que introduce el barrio no solo como un ámbito de estudio sino como una unidad de análisis que condiciona y explica el comportamiento móvil de sus residentes. Esto puede conllevar que, y como consecuencia del modelo barrial, existan residentes que dispongan de un alto grado de acceso, pero que, sin embargo, apuesten por un tipo de movilidad que no necesariamente implique ni grandes ni múltiples ni variados desplazamientos. Un ejemplo de ello serían las propuestas surgidas desde el nuevo urbanismo basadas en la configuración de vecindades compactas y con actividades diversas y cuyo objetivo, entre otros, es promover desplazamientos endógenos y peatonales (Shay et al. 2006) sin que ello signifique necesariamente una reducción del grado de opciones de movilidad ofertado. Esto puede generar en el residente un habitus 
ambulante aleccionado hacia la idea de acceder sin mover. Habitus diferente al de los que atesoran los habitantes de los suburbios de las ciudades dispersas cuyo acceso les orienta hacia el consumo de grandes trayectos en vehículos motorizados. Por tanto, un aspecto central a la hora de evaluar el capital de motilidad del que disponen los agentes no es solamente el número de trayectos o el grado de acceso del que dispongan sino también el nivel de satisfacción que expresen; es decir, si el medio residencial y sus posibilidades de movilidad se ajustan a sus intereses y estilo de vida.

Por todos estos motivos, este "capital territorial" se nos presenta sumamente sugerente e interesante por cuanto entreteje la movilidad, el transporte y el espacio en una misma unidad de análisis. No obstante, en nuestra opinión esa preponderancia dada al "capital territorial" para analizar los habitus ambulantes de los sujetos puede tener relevancia para el contexto latinoamericano analizado por el autor, donde las ciudades están experimentando un proceso acelerado y dramático de fragmentación y segregación socio-espacial; pero, ¿qué sucede con aquellos territorios barriales con un mismo grado de centralidad y acceso en los que todavía cohabitan clases sociales diferenciadas?, ¿cómo se explican entonces las diversas lógicas de movilidad de los residentes de diferentes clases a pesar de compartir un mismo hábitat?

En mi opinión lo que define sustancialmente ese capital, ese bien puesto en juego en el campo de la movilidad, no es otro que el propio desplazamiento. Tal y como nos sugieren Flamm y Kaufmann (2006) y Kaufmann, Bergman y Joye (2004), ese bien sería la posibilidad real o potencial de movilizarse, de llevar a cabo trayectos y viajes. Este ha de ser entendido, por tanto, como un capital por cuanto su acceso y su modo de interiorizar no solo son diferentes en función de la posición en el propio campo sino también desigual originando luchas por su control. Precisamente autores como Jouffe y Lazo (2010), Rodríguez (2008) o Cebollada y Avellaneda (2008) han enfatizado el carácter inequitativo en el acceso al sistema de transporte -en particular en América Latina- en función de componentes étnicos-raciales, económicos, de género y/o edad. Sin embargo, ese acceso desigual también genera y amplifica desigualdades constitutivas de otros campos. Fue Amartya Sen (2000) quien nos mostró que la pobreza no era producto solo de bajos ingresos económicos, sino fundamentalmente de la imposibilidad de desarrollar capacidades. Estas capacidades están emparentadas con la inserción dentro de los sistemas educativos-culturales, políticos y del mercado laboral, entre otros. Pensemos que para ejercer esos derechos es imprescindible apropiarse materialmente de esos sistemas: poder acceder a los centros de salud, a las escuelas, poder acudir a acciones políticas o al lugar de trabajo. Todos ellos requieren de desplazamientos físicos. Indudablemente una buena cobertura en términos de coste económico, tiempo invertido, seguridad y comodidad facilita esa movilidad y con ello el desarrollo de esas capacidades, así como la consumación de esos derechos básicos y la posible reducción de la brecha social existente.

Así pues, y como bien lo manifiesta Pierre Bourdieu, la configuración de un campo social está estrechamente relacionada con la presencia de un capital determinado, exclusivo y definitorio (1999). En esta ocasión en el campo de la movilidad se pondrá en liza el capital de motilidad. Para Kaufmann, Bergman y Joye 
(2004) entenderlo de este modo significa "que el movimiento puede adoptar muchas formas, que las diferentes formas de movimiento pueden ser intercambiables, y que la potencialidad del movimiento puede ser expresada en forma de 'capital de movimiento"' (2004, 752). Para estos autores ese capital está compuesto por aquellos tres aspectos diferenciadores ya mencionados: el grado de acceso, las competencias y la apropiación. El primero de ellos podría definirse del siguiente modo: son las limitaciones dadas que afectan a la movilidad en un territorio específico. Esas limitaciones pueden clasificarse en opcionales, las cuales se refieren a la variedad de medios de transportes, comunicación, servicios y equipamientos ofertados; y condicionales, que apuntan a la accesibilidad en cuanto al costo y frecuencia de los mismos. Cierto es que la accesibilidad dependerá tanto de la distribución de la población en el espacio, de las políticas públicas, como de la posición social de los individuos y grupos.

El segundo, el de las competencias, incluiría tanto las destrezas como las habilidades de los individuos y grupos. Estas destrezas y habilidades podrían dividirse a su vez en físicas, conocimientos adquiridos y habilidades en la organización estratégica de la movilidad.

Y por último la apropiación. Esta se refiere a la capacidad del individuo de interpretar su grado de movilidad a partir del acceso y sus competencias. Kaufmann, Bergman y Joye (2004) se refieren a ello cuando señalan la capacidad de interpretación que atesoran los individuos y grupos acerca del grado de acceso disponible y sus competencias, y conforme a ello actúan de un modo singular. Esta dimensión apunta claramente a los planes, aspiraciones, motivos, valores y hábitos, así como la valoración de las opciones de movilidad que experimentan y son protagonistas los propios sujetos. Por tanto, este aspecto se revela central en el análisis de la motilidad por cuanto articula las otras dos variables y permite indagar sobre la capacidad de agencia de los sujetos.

Retomando la noción de capital de motilidad, y al igual que cualquier otro tipo de capital, este también se pone en circulación: por ejemplo, con todas aquellas iniciativas gubernamentales de implantación de la movilidad a partir de la construcción de infraestructuras (red vial, de ferrocarriles, aeropuertos, red de ciclorrutas, puentes, semáforos: con políticas de comodalidad y conectividad a partir de la articulación de diferentes modos de transportes tales como autobuses, metros, tranvías, metrocables, trenes, aviones y/o vehículos no motorizados); desde el sector privado, el cual ofrece cada vez más la venta del propio artefacto (bicicletas, motocicletas, autos y otros dispositivos para el desplazamiento) y de servicios vinculados a la movilidad tanto en el ámbito local (con la conversión del modelo público de transporte colectivo a uno mixto o directamente privado; en particular nos referimos a la reconversión sufrida en la década de 1970 por el sistema público de buses de muchas ciudades en América Latina ${ }^{9}$ ), como en el nacional e internacional con la privatización de servicios tan emblemáticos y estratégicos como el del ferrocarril -casos de Bolivia y Brasil

9 Según Figueroa (2001), en la década de 1970 se consuma la desarticulación y desaparición de muchas empresas públicas de transporte que operaban en autobús. Algunas ciudades que contaban con este tipo de empresa eran México D.F., Santo Domingo, Caracas, Lima, La Paz, Santiago de Chile, Montevideo, São Paulo y Río de Janeiro. 
(Thompson 1997)- o de compañías aéreas de propiedad estatal, como las Aerolíneas Argentinas (Rey 2001); y, por último, desde las iniciativas populares como la red de vehículos privados proveedores de servicio público pero sin pertenencia a empresas del ramo (Jouffe y Lazo 2010).

Todo este despliegue de servicios y bienes de la movilidad espacial es consumido por los usuarios de un modo diferente y a menudo desigual. Ese consumo no solo estará mediado por la existencia o no del propio transporte, sino que también estará condicionado por el coste y la eficacia de este. Del mismo modo, la decisión de control del sistema de movilidad por parte de la administración (garantizando un grado de acceso óptimo para todas las capas sociales) o, por el contrario, la delegación en el diseño y su gestión en manos de empresas privadas condicionarán la acumulación (y con ello el volumen) de este capital por determinadas clases sociales. Pensemos en toda la inversión pública para aumentar y mejorar la red vial urbana de muchas ciudades de América Latina, cuando paradójicamente solo un porcentaje mínimo de su población es propietario de un vehículo privado motorizado -caso de la ciudad de Lima, en la que solo $17 \%$ es propietaria ${ }^{10}-$; o, al contrario, cuando las administraciones públicas deciden invertir en la configuración de una red de transporte colectivo tanto motorizado como no motorizado destinada principalmente a los sectores populares, como así lo demuestran los ejemplos de Quito y Curitiba con la implementación de un sistema de buses rápido (Pardo 2009). Como puede observarse, en algunos casos estas políticas inciden en la concentración del capital de motilidad, mientras que en otros en su redistribución.

Cuando estos procesos implican la pérdida del grado de motilidad ocasionando la insatisfacción de algunos de los agentes en el campo, esto puede conllevar luchas por controlar este capital. Estas pugnas pueden producirse en un nivel microsociológico, encarnándose, por ejemplo, en las maniobras, encontronazos o disputas que se despliegan en las calzadas de muchas ciudades latinoamericanas y en los que los usuarios en peor posición -es decir, ciclistas y peatones- son casi expulsados del espacio urbano y obligados a desplegar tácticas de supervivencia como puedan ser ir en grupos o utilizar acerados y andenes; o en un nivel macrosociológico con la pugna ya mencionada por controlar el campo de la movilidad entre organizaciones de ciclistas y de conductores. Lucha que no solo se circunscribe a un plano material, sino que posee su correlato también en lo discursivo: si los primeros acusan a los segundos de inmoralidad (Cupples y Ridley 2008), los conductores culpan a los ciclistas de problematizar el naturalizado tránsito de las vías. Igualmente, la movilidad puede transmutarse en un campo de luchas para otras dimensiones como la del sexo-género. Luis Vivanco (2013) describe que el montar en bicicleta se convirtió en un símbolo de las reivindicaciones de las mujeres sufragistas en la Inglaterra victoriana, puesto que utilizar este medio de transporte no solo les permitía un desplazamiento autónomo, sino también la adquisición de un nuevo habitus corporal expresado, por ejemplo, en el uso de una vestimenta que cuarteaba los estereotipos existentes en aquel tiempo. nal/2014/03/27/actualidad/1395931222_645507.html. 
Así pues, de esas decisiones dentro del campo, y que competen tanto a las estructuras sociales dadas como a los agentes mismos, estos acumularán o perderán parte de ese capital. En ese juego no solo factores exógenos influyen -pensemos en una mala planificación en la conectividad del sistema de transporte o en un colapso económico de las empresas privadas de determinado servicio de transporte ${ }^{\mathrm{I}}$-, también los endógenos desempeñan un papel relevante. Estos hacen referencia tanto al individual, cuando el sujeto pierde la oportunidad de uso del transporte y ello hace disminuir su capacidad de movilizarse, como al colectivo, cuando un segmento social es incapaz de situar en la arena política la movilidad espacial como un derecho social.

\section{La configuración de un habitus ambulante}

Ahora bien, la circunstancia de que el campo de la movilidad forme parte de intersecciones no es óbice para que este sea entendido como un mercado propio en el que aparezcan bienes y servicios diversificados en función de los diferentes perfiles de usuarios/clientes existentes. Estos productos son ofrecidos por agentes de producción e intermediación que cada vez se configuran de un modo más mixto, lo que moldea los diferentes habitus configurados en el campo de la movilidad. Por tanto, el modo de ser y estar en la vida social es fruto de la interiorización de las estructuras externas y de la posición dentro del campo, pero también de la capacidad de manipularlas a partir del despliegue de tácticas y estrategias; en definitiva, de la capacidad de agencia. En cualquier caso, el habitus proporciona al grupo o individuo un marco para disponer de representaciones de la realidad y de un repertorio de prácticas en sintonía con la propia posición que se ostenta.

Así pues, dentro de lo que podríamos denominar el campo de la movilidad el habitus de amplios sectores de la sociedad -en particular de las nuevas clases medias- ha encontrado, como consumidores de un marcado generoso, en la propiedad del vehículo motorizado no solo un dispositivo con el que satisfacer necesidades de desplazamiento, sino también un marcador de ascenso y adscripción de clase (Urry 2004; Sheller 2004). El auto, por tanto, es marcador de distinción no solo en términos simbólicos (de mostrar el objeto que externaliza el estatus), sino de práctica cotidiana. En efecto, a partir de un comportamiento diario de movilidad -conducir el auto, trasladarse por un mismo trayecto, poseer acceso a aparcamiento, recurrir a los servicios de limpieza específicos, adecuarlo estéticamente, participar en intercambios de servicios-, se podría aprehender una cultura de la movilidad (deudora de estructuras externas) interiorizada por un grupo (en esta ocasión por una nueva clase media emergente) y proyectada como imagen de pertenencia exclusiva de una clase por los miembros de esa misma clase. Obviamente, la configuración de un habitus ambulante no solo ha germinado en esas clases medias, también puede observarse en otros sectores

11 Un caso paradigmático es la gestión privada del sistema masivo de la ciudad colombiana de Cali en el que los problemas financieros de los operadores privados derivados de las fallidas expectativas de rentabilización del propio servicio están lastrando parte de su funcionamiento (http:// www.elpais.com.co/elpais/cali/noticias/estos-son-problemas-padecen-sistemas-transporte-masivo-pais. 05-03-2013). 
sociales, como así lo demuestran los estudios concentrados en el uso por parte de segmentos populares de las busetas y todo lo que conlleva de proceso de resocialización. Janacua y García (2013) analizan, desde la perspectiva de Erving Goffman, las interacciones sociales entre los usuarios de las combi en México -la mayoría de las clases subalternas- por cuanto este genera ciertos códigos tácitos de copresencia sui géneris que devienen, finalmente, en verdaderas marcas de identidad colectiva e individual.

Similar a este trabajo, encontramos con la investigación llevada a cabo por Horta y Malet (2014) sobre el hiace de la isla de Santiago del archipiélago de Cabo Verde. Viajar en este medio de transporte, parecido a la combi mexicana, instaura entre sus usuarios un modo de estar y ser en el desplazamiento que solo puede ser interiorizado en la medida en que se forma parte de esa lógica de movilidad. Lógica que podría sintetizarse en un proceso de continua negociación y aceptación de un tipo de funcionamiento que, bajo unas estructuras externas de precariedad, abarcan desde un sentido propio y reducido de la seguridad vial, una demanda constante de velocidad y un acomodo del usuario frente a la imprevisibilidad y cuyos efectos, según sus autores, se trasfieren a otras esferas de la vida cotidiana. En definitiva, en estos ejemplos podemos advertir la existencia y activación de un habitus ambulante particular que configura un estilo de vida, entendiendo por este un "sistema de prácticas enclasadas y enclasantes, esto es, de signos distintivos" (Bourdieu 2012, 201).

Por tanto, el campo de la movilidad -al igual que el campo económico, el religioso o el educativo- es una arena en la que están presentes pautas de acción irreflexivas, pero que obedecen a estructuras de pensamientos y acciones regladas en función de las posiciones sociales y de las formas de distinción delimitadas entre clases sociales. Es por ello que el de la movilidad también ha de ser considerado un espacio social idóneo donde observar y analizar esos hábitos de comportamientos, esas rutinas de desplazamiento, así como rastrear la existencia de un habitus. Y es en ese rastreo donde el cuerpo aflora como un elemento central en el que depositar la mirada analítica como consecuencia de su función en la ejecución de dichas acciones de apariencia presumiblemente maquinal, puesto que como bien lo sugiere Pierre Bourdieu:

\footnotetext{
El sentido práctico, necesidad social vuelta naturaleza, convertida en esquemas motrices y automatismos corporales, es lo que hace que las prácticas (...) sean sensatas, vale decir habitadas por un sentido común. Precisamente porque los agentes no saben nunca completamente lo que hacen, lo que hacen tiene más sentido del que ellos saben $(2008,111)$.
}

De este modo, la elección del modo de movilidad espacial es fruto tanto de un comportamiento automático y habitual como de la búsqueda de la optimización del coste del propio trayecto (Davidov 2007). Sin embargo, para evaluar esos hábitos de desplazamiento es imprescindible atender no solo a aspectos psicológicos, sino también a una serie de variables estructurales tales como las características socioeconómicas, educativas, de estatus, etaria, étnico-racial y de género, entre otras, que caracterizan al sujeto y lo sitúan en una posición social determinada, puesto que todas ellas inciden en sus preferencias hacia la elección del transporte y en la explicitación de un modo corporal de movilidad. La 
importancia de estas variables estructurales radica en que dotan al sujeto de una posición particular en el campo posibilitando su integración en una clase social determinada, así como la interiorización e incorporación de una serie de prácticas y representaciones sociales. Todo ello es lo que moldea el comportamiento móvil del individuo, lo que constituye, en mi opinión, su habitus ambulante.

\section{Conclusiones}

En suma, la movilidad socio-espacial además de ser considerada una necesidad social e individual es en sí misma un campo constituido por un capital propio que está estrechamente vinculado a los intereses de los actores sociales por cuanto es considerado socialmente un bien codiciado y escaso. Este capital, al igual que otros, es puesto en circulación -por tanto, es susceptible de acumulación o pérdida- en un campo donde los actores reconocen unas reglas y disponen de unos intereses. Como consecuencia de su carácter codiciado se establece una lucha por su control, puesto que la movilidad, o mejor, la posibilidad real o potencial de desplazarse (lo que hemos denominado motilidad) facilita la adquisición de otros capitales (o competencias en palabras de Amartya Sen (2000)) al situar al sujeto en una mejor posición social, al tiempo que le concede la oportunidad de que despliegue sus hábitos de movilidad.

Explicar esas rutinas de movilidad, esos comportamientos de desplazamientos diarios de los individuos, y también las representaciones que poseen sobre los medios de transporte, nos remite inevitablemente a la noción de habitus, el cual es el dispositivo agregado de percepciones y prácticas que caracteriza al sujeto siendo producto de la posición social que este ocupa al tiempo que da cuenta del modo en el que ha interiorizado esas mismas estructuras externas. Así pues, este habitus ambulante estará conformado por las prácticas cotidianas de movilidad, es decir, las rutas seleccionadas, los dispositivos de desplazamientos utilizados, la interacción que establecen los individuos con esos mismos dispositivos y con otros individuos; pero también formarán parte de este habitus, tal y como ya se enunció, las representaciones que poseen los sujetos acerca de esos recorridos, de la propia noción de movilidad así como de los medios de transporte disponibles y, en última instancia, del grado de satisfacción con respecto a su movilidad y a las políticas de movilidad socio-espacial promovidas.

\section{Referencias bibliográficas}

Acevedo, Jorge, Juan Bocarejo, Juan Echeverry, Germán Lleras, Germán Ospina y Álvaro Rodríguez. El transporte como soporte al desarrollo de Colombia. Una visión al 2040. Bogotá: Uniandes, 2009.

Almaraz, José, Julio del Pino, Juan Izquierdo, Francisco García, Alberto Cillero, Paula Bouzada, Juan Jiménez, Ángeles Táuler, Víctor Sánchez, María Lloret y María Sospreda. «Transporte y elección modal. Aplicaciones de sociología de la movilidad». Ponencia presentada en el $X$ Congreso Español de Sociología, Pamplona, España. 30 de junio - 3 de julio de 2010. Disponible en http:// www.fes-sociologia.com/gt-5-sociologia-urbana/pages/155/ (último acceso: 24 de septiembre de 2014). 
Beck, Ulrich. World Risk Society. Cambridge: Polity Press, 1999.

Bericat, Eduardo. Sociología de la movilidad espacial. El sedentarismo nómada. Madrid: Siglo XXI-CIS, 1994.

Blickstein, Susan, and Susan Hanson. «Critical Mass: Forging a Politics of Sustainable Mobility in Age Information". Transportation, Vol. 28, $\mathrm{n}^{\circ}$ 4, 2001: 347-362.

Bourdieu, Pierre. Razones prácticas sobre la teoría de la acción. Barcelona: Anagrama, 1999.

Bourdieu, Pierre. El sentido práctico. Madrid: Siglo XXI, 2008.

Bourdieu, Pierre. La distinción. Criterios y bases sociales del gusto. México D.F.: Tauro, 2012.

Canzler, Weert, Vincent Kaufmann, and Sven Kesselring. «Tracing Mobilities. An Introduction». In Tracing Mobilities. Towards a Cosmopolitan Perspective, edited by Weert Canzler, 1-10. Farnham: Ashgate, 2008.

Castells, Manuel. La cuestión urbana. Madrid: Siglo XXI, 1974.

Cebollada, Ángel y Paul Avellaneda. «Equidad social en movilidad: reflexiones en torno a los casos de Barcelona y Lima». Scripta Nova. Revista Electrónica de Geografía y Ciencias Sociales, Vol. XII, n 270 (47), 2008. Disponible en http:// www.ub.edu/geocrit/sn/sn-270/sn-270-47.htm (último acceso: 18 de octubre de 2014).

Cupples, Julie, and Elisabeth Ridley. «Towards a Heterogeneous Environmental Responsibility: Sustainability and Cycling Fundamentalism». Wiley, Vol. 40, $\mathrm{n}^{\mathrm{O}} 2$ (June 2008): 254-264.

Davidov, Eldad. «Explaining Habits in a New Context: The Case of Travel-Mode Choice». Rationality and Society, Vol. 19, $\mathrm{n}^{\circ}$ 3, 2007: 315-334.

Elliott, Anthony, and John Urry. Mobilities Lives. Nueva York/Londres: Routledge, 2010.

Figueroa, Oscar. «Políticas de desarrollo y políticas de transporte urbano. Coherencias y contradicciones». En La ciudad construida. Urbanismos en América Latina, de Fernando Carrión, 377-390. Quito: Flacso, 2001.

Flamm, Michael, and Vincent Kaufmann. "Operationalising the Concept of Mobility: A Qualitative Study". Mobilities, Vol. 1, n² 2, 2006: 167-189.

Furness, Zack. "Critical Mass, Urban Space and Velomobility». Mobilities, Vol. 2, $\mathrm{n}^{\circ}$ 2, 2007: 299-319.

Greenfield, Adam. Against the Smart City. s.c.: Kindle Edition, 2013.

Gutiérrez, Alicia. Las prácticas sociales: una introducción a Pierre Bourdieu. Córdoba: Ferreyra Editor, 2005.

Harvey, David. La condición de la posmodernidad: Investigación sobre los orígenes del cambio cultural. Buenos Aires: Amorrortu Editores, 1998.

Horta, Gerard y Daniel Malet. Hiace. Antropología de las carreteras en la isla de Santiago (Cabo Verde). Barcelona: Pol.len Edicions, 2014.

Janacua, Jesús y Raúl García. «Interacción social y transporte público: Erving Goffman en la combi». URBS. Revista de Estudios Urbanos y Ciencias Sociales, Vol. 3, $n^{\circ}$ 1, 2013: 144-154.

Jenssen, Mette. «Passion and Heart in Transport - a Sociological Analysis on Transport Behaviour». Transport Policy, nº 6 (1999): 19-33. 
Jouffe, Yves. «Las clases socio-territoriales entre la movilidad metropolitana y el repliegue barrial. ¿Tienen los pobladores una movilidad urbana de clase?». Revista Transporte y Territorio, $\mathrm{n}^{\circ}$ 4, 2011: 84-117.

Jouffe, Yves y Alejandra Lazo. «Las prácticas cotidianas frente a los dispositivos de la movilidad. Aproximación política a la movilidad cotidiana de las poblaciones pobres periurbanas de Santiago de Chile». EURE XXXVI, $\mathrm{n}^{\circ}$ 108, 2010: 29-47.

Kaufmann, Vincent, Manfred Bergman, and Dominique Joye. «Motility: Mobility as Capital». International Journal of Urban and Regional Research, Vol. 24, $\mathrm{n}^{\circ}$ 4, 2004: 745-756.

Kesselring, Sven. «The Mobile Risk Society». In Tracing Mobilities. Towards a Cosmopolitan Perspective, edited by Weert Canzler, Vincent Kaufmann, and Sven Kersserling, 77-101. Farnham: Ashgate, 2008.

Marqués, Juan. «El mundo por delante». En Caminar, de William Hazlitt y Robert Louis Stevenson, 13-21. Salamanca: Nordica Libros, 2015.

Pardo, Carlos. Los cambios en los sistemas integrados de transporte masivo en las principales ciudades de América Latina. Santiago de Chile: CEPAL, 2009.

Rey, Mabel. Alas rotas. La política de privatización y quiebra de Aerolíneas Argentinas. Buenos Aires: Tema, 2001.

Rodríguez, Jorge. «Movilidad cotidiana, desigualdad social y segregación residencial en cuatro metrópolis de América Latina». EURE, Vol. XXXIV, ${ }^{\circ} 103,2008$ : 49-71.

Sen, Amartya. Desarrollo y libertad. Bogotá: Planeta, 2000.

Shay, Elizabeth, Yingling Fan, Daniel Rodríguez, and Asad Khattak. "Drive or walk? Utilitarian Trips within a Neotraditional Neighborhood». Journal of the Transportation Research Board, $\mathrm{n}^{\circ}$ 1985, 2006: 154-161.

Sheller, Mimi. «Automotive Emotions: Feeling the Car». Theory, Culture \& Society, Vol. 21, $\mathrm{n}^{\circ}$ 4-5, 2004: 221-242. Available in http://www.comp.lancs.ac.uk/ sociology/soc124ms.pdf (accessed: 12 de febrero de 2014).

Thompson, Ian. Los ferrocarriles y su contribución al comercio internacional. Buenos Aires: Banco Interamericano de Desarrollo-INTAL, 1997.

Ureta, Sebastián. «Manejando por Santiago. Explorando el uso de automóviles por parte de habitantes de bajos ingresos desde una óptica de movilidad sustentable». Revista Eure, Vol. XXXV, n 115, (2009): 71-93.

Urry, John. Sociology Beyond Societies. Mobilities for the Twenty-first Century. Londres: Routledge, 2000.

Urry, John. «The System of Automobility». Theory, Culture \& Society, Vol. 21, ${ }^{\circ}$ 4/5, 2004: 25-39.

Virilio, Paul. La velocidad de liberación. Buenos Aires: Manantial, 1997.

Vivanco, Luis. Reconsidering the bicycle. An anthropological perspective on a new (old) thing. New York/London: Routledge, 2013. 